\title{
Image analysis of real-time classification of cherry fruit from colour features
}

\author{
José F. Reyes, Elías Contreras, Christian Correa, Pedro Melin \\ Faculty of Agricultural Engineering, Universidad de Concepción, Chillán, Chile
}

\begin{abstract}
An image analysis algorithm for the classification of cherries in real time by processing their digitalized colour images was developed, and tested. A set of five digitalized images of colour pattern, corresponding to five colour classes defined for commercial cherries, was characterized. The algorithm performs the segmentation of the cheery image by rejecting the pixels of the background and keeping the image features corresponding to the coloured area of the fruit. A histogram analysis was carried out for the RGB and HSV colour spaces, where the Red and Hue components showed differences between each of the specified colour patterns of the exporting reference system. This information led to the development of a hybrid Bayesian classification algorithm based on the components $\mathrm{R}$ and $\mathrm{H}$. Its accuracy was tested with a set of cherry samples within the colour range of interest. The algorithm was implemented by means of a real time $\mathrm{C}++$ code in Microsoft Visual Studio environment. When testing, the algorithm showed a $100 \%$ effectiveness in classifying a sample set of cherries into the five standardized cherry classes. The components of the hardware-software system for implementing the methodology are low cost, thus ensuring an affordable commercial deployment.
\end{abstract}

\section{Introduction}

An increasingly important aspect in the agribusiness is the inclusion of automatic selection and sorting equipment in fruit and vegetable processing lines (Moreda et al., 2009; Kondo, 2010). This trend towards automation is due to the decreasing availability of labour, and, on the other hand, their inadequate level of training, which directly affects the quality of the final product.

Correspondence: José F. Reyes, Faculty of Agricultural Engineering, Universidad de Concepción, Casilla 537, Chillán/Chile. E-mail: jreyes@udec.cl

Key words: Colour sorting; cherries; fruit automation.

Received for publication: 9 February 2021

Accepted for publication: 8 July 2021.

${ }^{\circ}$ Copyright: the Author(s), 2021

Licensee PAGEPress, Italy

Journal of Agricultural Engineering 2021; LII:1160

doi:10.4081/jae.2021.1160

This article is distributed under the terms of the Creative Commons Attribution Noncommercial License (by-nc 4.0) which permits any noncommercial use, distribution, and reproduction in any medium, provided the original author(s) and source are credited.
Likewise, their low efficiency and slowness leads to an increase in costs (Singh et al., 1993). Moreover, manual selection of fruits, especially based on colour, requires a high degree of discrimination in a range of shades, a skill that humans cannot always offer. The human visual system can correctly perceive the colour of objects regardless of light conditions (Ebner, 2004). Indeed, the light intensity range to which the human eye can adapt is the order of $10^{10}$ miliambert between the scotopic perception threshold and the glare limit (González and Woods, 2006). However, it has little ability to repeat its quality estimation (Paulus et al., 1997), which can vary from one worker to another and depends their mood. The colour distributed homogeneously on the surface of a fruit, called primary, when averaged, becomes a good indicator of their quality (Blasco et al., 2003) and has a key role in the quality assessment by the consumer (Antonelli et al., 2004). Therefore, in most processing plants a selection process is performed by colour. In the case of cherries, this process is mainly performed by manual selection. However, this task can also be performed by some equipment, which can be based on various operating principles and processing capabilities, with unattainable prices for small producers. In the local Chilean market, some types of equipment in use perform selection by colour and are still based on a technology from the nineties, which is insufficient to increase processing capabilities. Another drawback is that these systems can no longer benefit from technical support because they are too old and manufacturers, which are based in the EU and US, find it difficult to keep up with maintenance and repair requirement. A feasible solution is the implementation of algorithms to upgrade existing equipment, therefore using the same hardware, but adding some more efficient software from the computational point of view.

Fruit and vegetable classification methods have been developed for quality assessment and robotic harvesting, but so far has been developed for limited classes and small datasets. The problem is of a multi-dimensional nature since involves three colour components, which is one of the major challenges with current machine learning approaches. Substantial research has been conducted to design and analyze classifiers of this type which require significant computational power to optimize such features. In recent years numerous machine learning techniques such as, for example, support vector machine (SVM), K-nearest neighbour (KNN), decision trees, artificial neural networks (ANN) and convolutional neural networks (CNN) have been exploited with many different feature description methods for fruit and vegetable classification in many real-life applications (Khurram et al., 2018). There are several algorithms using techniques of supervised machine learning (Kotsiantis, 2007), that can be used for classifying fruits using colour, size, shape, and texture (Funck et al., 2003; Zheng et al., 2006; Arivazhagan et al., 2010). Machine vision for grading fruits has been applied both in the visible and the infrared region (Lee et al., 2008; Rungpichayapicheta et al., 2016; Zhang et al., 2016). Singh et al. (1993) used a digitized photograph of a fruit, numbering the pixels belonging to each of the 13 colour references available, and rating the fruit based on the kind of colour of the pixels, if they accounted at least for $20 \%$ of the total pixels. 
The results with this technique improve by $10 \%$ compared to manual selection. Ahmad and Reid (1996) included RGB and HSI colour spaces in their classification technique to determine variations in the levels of nitrogen and water in corn husks. The results were not optimal due to the variable of the operator subjective rating, however the spaces used made it possible to distinguish small variations in tone. Further research was directed to specific applications. For instance, Paulus et al. (1997) selected apples into two categories (yellow or green) and separated them by a bimodal histogram analysis, thresholding with functions that combine RGB value percentages. Antonelli et al. (2004) used colour-grams, which are matrices with mean coordinates in red, green, blue (RGB) and hue, saturation intensity (HSI) digital spaces, plus a set of parameters derived there from, obtaining an effectiveness of $100 \%$ in the recognition of similar object textures. There is also research aimed to identify multiple classes simultaneously, based on fuzzy classification algorithms (Correa et al., 2012). Such methods, despite their good results, have high requirements in terms of computing execution time. The Bayesian methodology to distinguish among classes has been used widely for the purpose of fruit and vegetable classification, particularly in real time applications due to its fast resolution. Blasco et al. (2003) presented a technique based on a Bayesian classifier using the components of the digital RGB colour space to recognize fruit size (apples, oranges, and pears) and damage and achieved an effectiveness of $86 \%$ and $93 \%$ respectively. A similar method was proposed by Rahimizadeh et al. (2009) that used a decision boundary equation, acquired from class-conditional probability density function, based on Bayes theorem. Gan et al. (2018) developed a methodology for green citrus fruit detection using fusion of colour and thermal images. Tan et al. (2018) recognized blueberry fruit with a different maturity using histogram-oriented gradients and colour features in outdoor scenes. Abdelghafour et al. (2019) used a Bayesian framework for joint structure and colour based pixelwise classification of grapevine proximal images. Wu et al. (2019) studied the recognition of peaches on trees combining 3D contour features and colour data. One of the last pieces of research on fruit recognition was conducted by Kang et al. (2020) who implemented segmentation and $3 \mathrm{D}$ visualization of environments in apple orchards using deep neural networks. Specifically, for cherries, a study was carried out with the aim of developing cherry fruit packing methods using the improved $\mathrm{CNN}$ algorithm to detect the appearance of cherries and provide an efficient system for their grading (Momeny et al., 2020).

This study uses image processing algorithms with low computational requirements, which are reliable from the application point of view, robust in operation and with quick technical support in case of technical problems. The main aim is to implement a combination of low computational and hardware requirements, such as a simple RGB camera, a desktop conventional computer and subsequently a low-cost real-time classification system of cherries in a packaging line. This paper is based on the use of a Bayesian classifier, which is a classification method characterized by the development of class discriminator functions built with known probability distributions, where the statistical average and standard deviation parameters are estimated for each class.

\section{Materials and methods}

\section{Methodology sequence}

The experimental procedure involved the following steps: i)
Image acquisition with a colour CCD camera and a digitizing device under constant artificial illumination conditions. To develop the algorithm, 63 samples of cherries having a colour within the classification range were analysed; ii) Image segmentation and data conversion from colour space RGB (red, green, blue) to HSV (hue saturation value); iii) Searching and capturing statistical parameters from RGB and HSV colour spaces to identify discrimination parameters among the five colour categories utilized to classify cherries; iv) Creation of comparison vectors, or discriminatory functions, using probability density functions for each of the five colour categories, which were built with the space colour components that showed the maximum discrimination power (Bayesian classifier); v) Assessment of the proposed algorithm from the accuracy and speed point of view. Accuracy was evaluated using 36 cherries, with colour within the range of the five categories of interest. These samples were generated independently from those used to develop the algorithm, but they were captured under the same illumination conditions. The colour grading standards were supplied by the Chilean Company Viverosur Ltd. and are shown in Figure 1. This colour classification is a commercial standard tool to perceive the degree of ripeness and acceptance by the consumer. Time was measured in the real-time computer code and compared with the time usually reported for equivalent manual operations.

\section{Experimental setup}

A fruit conveying experimental system was implemented to capture cherry images from a white moving belt, simulating a realworld classification system as shown in Figure 2. Image capture was performed with a CCD colour camera Model HD-31/A, NTSC format, of 5,120,000 pixels and shooting speed of 1/1000 s. These images were captured in BMP (bitmap) uncompressed format, using 24 bits, 8 for each component. Digitalization was performed with a card from PHOEBE, Model TV Master + FM, with a maximum capture rate of 30 frames per second and a maximum reso-

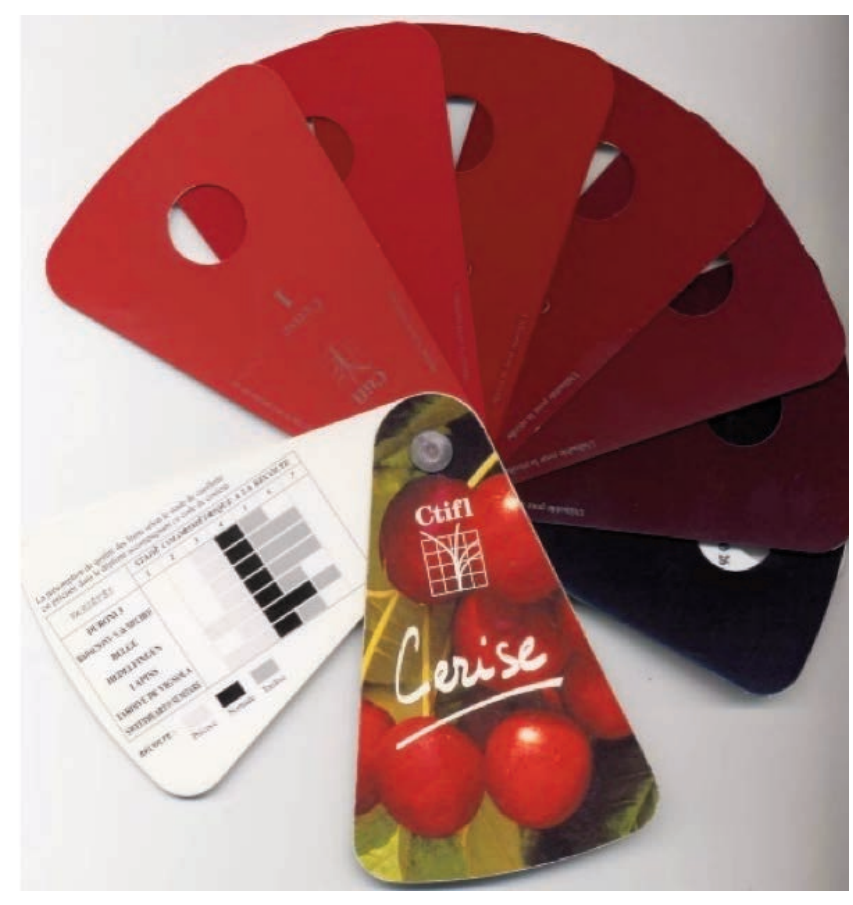

Figure 1. Grading colour standard of exportable cherries. 
lution of 640x480 pixels, which is compatible with AVI format. The capturing card was installed in a PC with a K6-II processor running at $300 \mathrm{MHz}$ and with $164 \mathrm{Mb}$ of RAM, where the computer code was developed and tested. The illumination setup was intended as to achieve a multi-directional diffuse light to minimize shadows and avoid excessive brightness. A fluorescent $32 \mathrm{~W}$ lamp energized by means of a $35 \mathrm{KHz}$ ballast was used. The ballast was installed to modify the low $50 \mathrm{~Hz}$ original frequency of the $220 \mathrm{~V}$ mono phase service, which causes undesirable illumination noise to the camera sensor.

\section{Image segmentation}

The most frequently used colour models used in image processing for colour recognition are the RGB and HSI spaces (González and Woods, 2006). The RGB colour space is the simplest to use and permits fast processing for real time applications, when using segmentation computer algorithms (Sowmya and Sheelarani, 2009). For the purpose of segmenting the area corresponding to the fruit of interest, with respect to the background area of the image, a double thresholding methodology was chosen in the primary colour space RGB, in order to obtain a segmentation as fast as possible, for real time implementation of the algorithm. Segmentation corresponding to the fruit area, with respect to the background of the image, can be expressed by a thresholding algorithm for each pixel $I(i, j)$ of the captured image:

$I(i, j)=\left\{\begin{array}{l}\text { fruit if } \quad G_{i j} \leq G_{t g} \cap B_{i j} \leq B_{t b} \\ \text { background if } G_{i j}>G_{t g} \cup B_{i j}>B_{t b}\end{array}\right\}$

where:

$G_{t b}$ y $B_{t b}$ are the thresholding values to which the components $\mathrm{G}$ and $\mathrm{B}$ of pixel $I(i, j)$ are compared with. Once the pixels of the fruits were separated from the rest of the image, a statistical Bayesian classifier based upon discriminate function was applied to label the fruit to one of the five classes.

\section{Discriminant functions}

The RGB colour space uses a Cartesian system, where each colour is formed with three spectral components from the visible light spectrum, between 400 and $700 \mathrm{~nm}$ of wavelength red, green, and blue. A more perceptual manner of representing the colour to the human eye is in terms of components: saturation $(\mathrm{S})$, hue $(\mathrm{H})$ and intensity or value (V), known as HSV space. The utility of this colour representation comes from to the fact that the intensity component $\mathrm{V}$ is decoupled from the chromatic information. The chromatic components hue $(\mathrm{H})$ and saturation $(\mathrm{S})$ are closely related to how the human beings perceive the colour. The intensity incorporates a chromatic notion of brightness, while the hue component is an attribute associated to the dominant wavelength among a blend

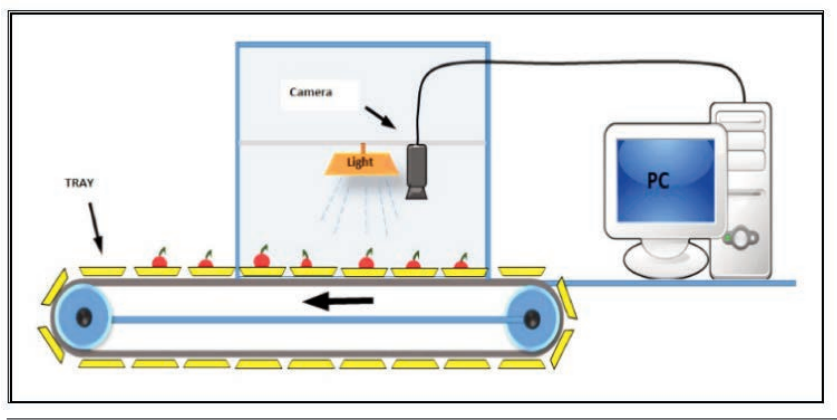

Figure 2. Image capture and processing system. of light wavelengths. Saturation is referred as to the relative weight of the dominant colour, or the amount of white colour mixed with the dominant colour. The combination of saturation and hue is known a chromaticity. Therefore, a colour may be characterized by means of its intensity and chromaticity. In this study, to assess the discriminatory behaviour of each of the colour components of the fruit, a histogram analysis for each of the components in RGB and HSV colour spaces was carried out. A histogram of a digital image with grey levels in the range [0, L-1] based on a discrete function $P\left(r_{l}\right)=n_{l} / n$, where $r l$ is the $l$-th grey level, $n_{l}$ is the number of pixels in the image with $l$-th grey level, $n$ is the total number of pixels in the image and $l=0,1,2, \ldots, \mathrm{L}-1$. The function $P(r l)$ gives an estimation of the probability of occurrence of the $r$ grey level [10]. This concept is valid for the statistical analysis of each of the components of RGB and HSV colour spaces. If the data is grouped into $j$ classes as in a histogram with $k=255$, all the values of each class are identified with the centre of the class $C_{j}, j=1,2, \ldots, k$. In this case the arithmetic mean is written as:

$$
\bar{x}=\sum_{j=1}^{k} C_{j} f_{j}
$$

where:

$f_{j}$ is the frequency of the $j$ class.

The definition of standard deviation for the data grouped into $k$ classes is expressed as:

$$
s=\sqrt{\sum_{j=1}^{k}\left(C_{j}-\bar{x}\right)^{2} f_{j}}
$$

After the assessment of these parameters for each of the RGB and HSV space components, the most statistically significant components were identified to discriminate between reference classes defined by quality commercial standards. The Bayesian classifier is based upon a statistical hypothesis of the behaviour of the classes, when the measures of dispersion of the classes are statistically significant. In class recognition, the Bayesian classifier is recommended as a highly reliable statistical tool (Leemans et al., 2002). If a normal or Gaussian one-dimensional distribution is considered for the discriminating functions, then the probability function for the Bayesian classifier can be written as follows:

$$
p\left(X / C_{j}\right)=\frac{1}{\sqrt{2 \pi \sigma_{j}^{2}}} \exp -\left[\frac{1}{2} \frac{\left(x-m_{j}\right)^{2}}{\sigma_{j}^{2}}\right]
$$

where:

$C_{1}, C_{2}, \ldots$ are the different classes, $m_{j}$ and $\sigma_{j}$ are the mean and the standard deviation respectively for the $X$ parameter in the $C_{j}$ class. In the case of a Gaussian probability density function that includes more than one parameter $X$, which doesn't correlate with the others, the Gaussian distribution is given by:

$p\left(X / C_{j}\right)=\prod_{i=1}^{p} \frac{e^{-\frac{1}{2}}\left(x_{i}-m_{j}\right)^{2}}{\frac{1}{\sqrt{2 \pi \sigma_{j i}^{2}}}}$

where:

$p$ is the number of characteristics of $j$ class, $m_{j l}$ to $m_{j p}$ are the means of the $x_{1}$ to $x_{p}$ characteristics for the $C_{j}$ class, while $\sigma_{j 1}$ and $\sigma_{j p}$ are their standard deviations respectively. In this research work, the classification method used a probability function as the 
Equation (5), where a minimal number of characteristics necessary to achieve a robust classification were identified. After identifying these colour characteristics, a vector $X^{*}$ containing the value of the mean of each characteristic must be defined as:

$\bar{x}=\sum_{j=1}^{k} C_{j} f_{j}$

where:

$X_{1}, X_{2}, X_{3}, \ldots X_{p}$ are real numbers, representing the value of each discriminator parameter utilized in the classification. The calculated vector $X$ was then compared to the probability functions, determined for each of the classes previously defined, to discriminate to which of the classes the fruit belongs to, depending on the function that gives the higher value of probability. To identify the discriminating colour components, a statistical analysis including the assessment of mean and standard deviation was performed for a set of 63 representative samples of cherries pertaining to the colour range. Statistical analysis was applied to each of the components of the RGB and HSV colour spaces, similarly to the work done by Paulsson and Stocklassa (1999) to find attributes in the HSV colour space. Once the main discriminating components were found, Equations (5) and (6) were implemented to determine the probability functions. In this case, the $\mathrm{R}$ and $\mathrm{H}$ components showed to be the more representative colour parameters to build the probability functions:

$p\left(X / C_{j}\right)=\frac{e^{-\left\{\frac{1}{2}\left[\frac{\left(R_{S}-m_{i R}\right)^{2}}{\sigma_{i R}^{2}}+\frac{\left(H_{S}-m_{j H}\right)^{2}}{\sigma_{i H}^{2}}\right]\right\}}}{2 \pi \sigma_{i R} \sigma_{i H}}$

$\boldsymbol{X}=\left[\begin{array}{l}R_{s} \\ H_{s}\end{array}\right]$

where:

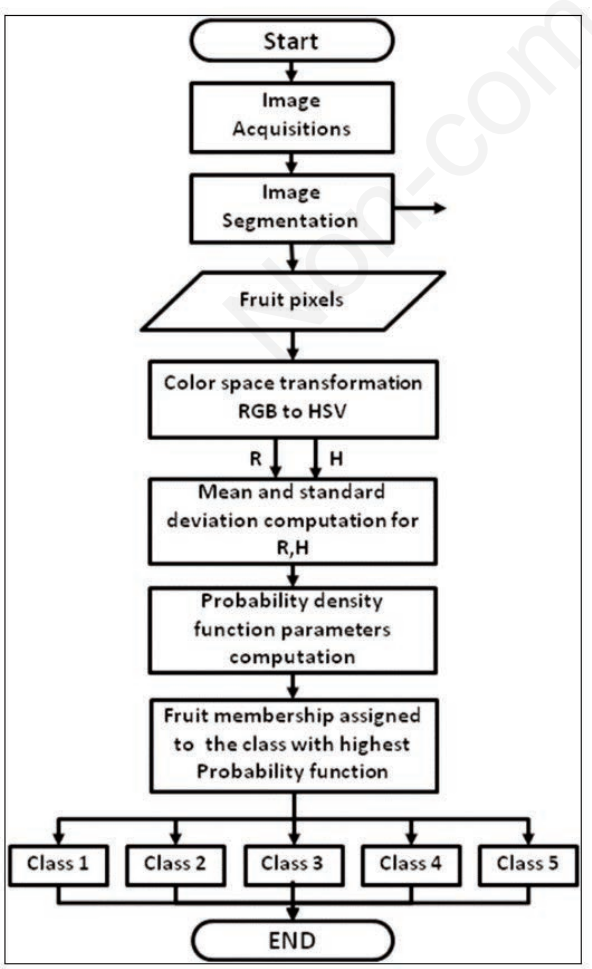

Figure 3. Flow sheet of the computer program.
$R_{S}$ and $H_{S}$ are the values of the mean of the fruit $\mathrm{R}$ and $\mathrm{H}$ components to classify; $m_{j R}, \sigma_{j R}$ and $m_{j H}, \sigma_{j H}$ are the mean and standard deviation of the $\mathrm{R}$ and $\mathrm{H}$ components of each class respectively, with $j=1$ to 5 classes.

\section{Computer codes}

The structure of this computer code is illustrated in Figure 3. In order to implement the real time classification methodology, two computer routines were developed in $\mathrm{C}++$ code. The first code performed the fruit segmentation and the statistical analysis, average and standard deviation of $\mathrm{R}, \mathrm{H}$ parameters, for 63 samples of cherries pertaining to five classes. This routine made it possible to create the discrimination probability functions according to Equation (7), necessary to classify statistically each fruit within five classes. A second computer code was developed for processing and classification of cherries in real time. This program loads in real time the fruit image taken under the same conditions as the reference patterns. Then, the routine proceeds with the segmentation of the fruit pixels from the the image background, using the threshold algorithm of the Equation (1). The following step is the extraction of the discriminating $\mathrm{R}$ and $\mathrm{H}$ components of the segmented fruit and the construction of the feature fruit vector. Then, with these values of $\mathrm{R}$ and $\mathrm{H}$, the discriminating functions are evaluated for the five classes according to Equation (7) and the one with the highest probability value of the sample is chosen. The evaluation of the algorithm was done using a series of 36 cherries that were chosen randomly within the range of five colour pattern classes and classified into one class for the program. With respect to the processing time required by the computer program, it was calculated internally, thus providing an estimate of the amount of fruit to be processed per minute, in order to be able to compare it with the average amount that is able to process a trained manual operator over the same period of time. The flow diagram of the computing algorithm is showed in Figure 3.

\section{Results and discussion}

Figure 4 reports the values of the mean versus the standard deviation for the R, G, B and $\mathrm{H}$ components of the 63 samples, corresponding to the five classes. Figure $4 \mathrm{~A}$ and $\mathrm{D}$ clearly show how the mean and the standard deviation of the $\mathrm{R}$ and $\mathrm{H}$ components permit a higher degree of discrimination between classes. Besides,

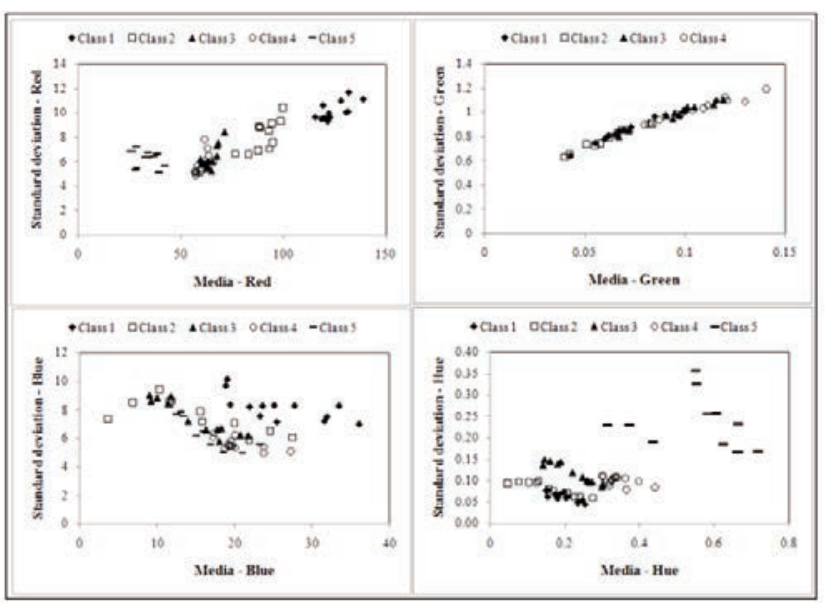

Figure 4. Standard deviation versus mean for Red, Green, Blue and Hue components of fruit samples. 
Figure 4A also shows that there is an overlapping for classes 3 and 4 with respect to the $\mathrm{R}$ component, while Figure 4D reveals an overlapping for the $\mathrm{H}$ component among the classes 1, 2 and 3. This information shows that, since the probability functions contain characteristics of other classes, they can potentially enhance the quality of the classification. The analysis showed in Figure 4 made it possible to select the $\mathrm{R}$ and $\mathrm{H}$ components to build the discriminating probability functions. These functions evaluated for 63 reference samples are depicted in Figure 5. A typical result of the segmentation with the real time code is shown in Figure 6. The next step was the extraction and average of the $\mathrm{R}$ and $\mathrm{H}$ fruit components to build the vector of characteristics defined in Equation (8). Finally, the vector of characteristics is used to evaluate the five probability functions according to Equation (7) and to label the fruit within the class with higher probability function value.

Moreover, the classification procedure in real time schematically shown in the flowchart in Figure 3 was implemented computationally in $\mathrm{C}++$ language using Microsoft Visual Studio as development environment. For each experimental sample, the computer program performed initially the fruit segmenting to detect the useful area of the sample to be classified. This initial segmentation was designed using thresholds for the Green and Blue components of the RGB space as shown in Equation (1), which made it possible to discriminate the area of interest from the image. Regarding the effectiveness of the classification, Table 1 shows the behavior of the algorithm of digital color selection of 36 random cherries, by means of a confusion matrix, where the number of samples classified within each pattern is indicated.

Table 1 shows the behaviour of the algorithm for the digital colour selection of 36 random cherries by means of a confusion matrix, where the number of samples classified within each pattern is indicated. The algorithm was implemented and tested on two processors from different processing speeds. In the case of a computer with a processor speed of $300 \mathrm{Mhz}$, the processing time varied between 442 and $471 \mathrm{~ms}$ with an average of $457 \mathrm{~ms}$ and a standard deviation of $7.7 \mathrm{~ms}$. While for a computer of $2.7 \mathrm{GHz}$, this time ranged between 240 and $270 \mathrm{~ms}$ with an average of $251 \mathrm{~ms}$ and a standard deviation of $8.0 \mathrm{~ms}$. The algorithm showed an effectiveness of $100 \%$, probably since sample tones were close to each pattern. Although there is a degree of overlap between the classes, this result means that the selected features are reliable to obtain a robust discrimination. Moreover, as regards the speed of the algorithm, the average classification time indicates that it is possible to process approximately 4 fruits per second $(100 \mathrm{~kg} / \mathrm{h})$ in a process line. This is comparable to the speed of a trained person considering that in general a line of cherry selection with 6 to 10 people has typically a yield of 1 ton per hour. These results show that this approach is a viable alternative to be implemented in agro-industrial plants, where premium cherries need to be classified by quality. However, it is important to take into account that it is possible to improve the processing speed by both optimizing algorithm and by using higher speed processors. The robust evaluation of the specific colour class of the cherries allows to estimate the level of ripeness and therefore the optimal consuming qualities of this fruit.

\section{Conclusions}

The implemented algorithm based on the Bayesian methodology, along with an off-the-shelf hardware, made it possible to perform an optimal classification of cherries in real time to meet international fruit quality standards. The experimental machine vision system used low-cost conventional devices and technologies available worldwide and at the regional level. The positive experimen-

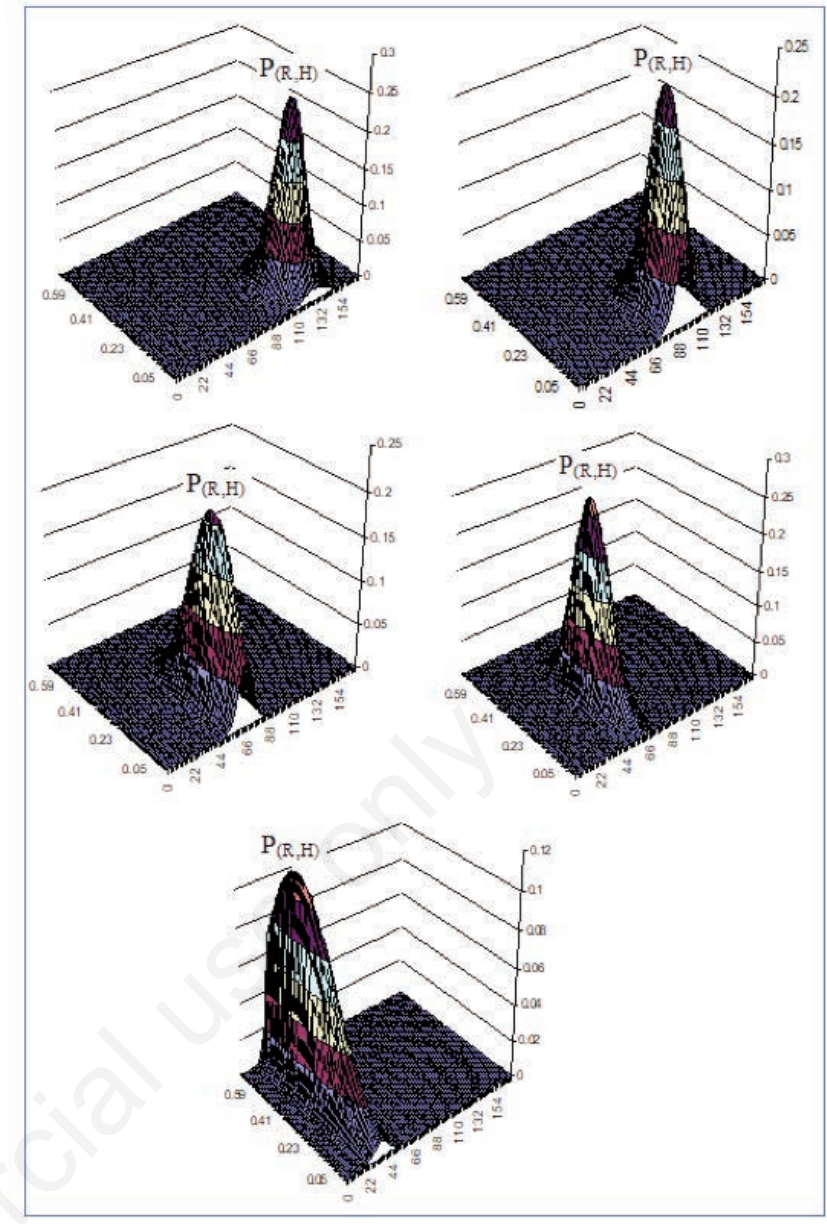

Figure 5. Gaussian probability density functions $P(R, H)$ for the five classes.

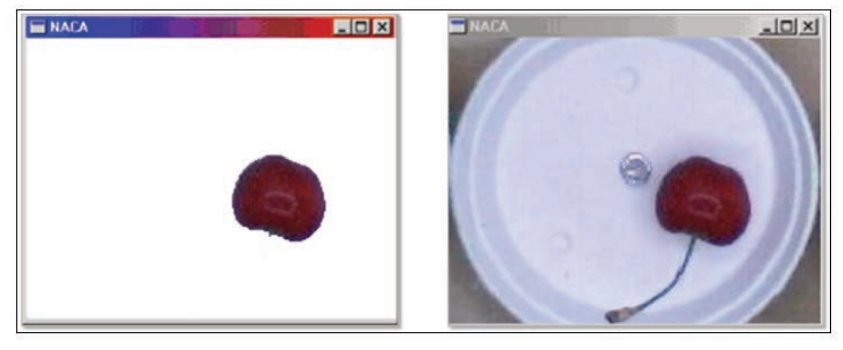

Figure 6. Typical sample of segmentation displayed by the computer code.

Table 1. Matrix of confusion with results from the classification algorithm testing.

Class 1 Class 2 Class 3 Class 4 Class 5 Total

\begin{tabular}{lllllll} 
Class 1 & 7 & 0 & 0 & 0 & 0 & 7 \\
Class 2 & 0 & 7 & 0 & 0 & 0 & 7 \\
\hline Class 3 & 0 & 0 & 9 & 0 & 0 & 9 \\
Class 4 & 0 & 0 & 0 & 6 & 0 & 6 \\
\hline Class 5 & 0 & 0 & 0 & 0 & 7 & 7 \\
Total & 7 & 7 & 9 & 6 & 7 & 36 \\
\hline
\end{tabular}


tal results enabled us to conclude that upgrading this conceptual technology up its practical implementation is feasible with basic technical capabilities, which would offer enormous advantages in terms of cost-capacity ratio to the local fruit processing companies. It is fundamental to emphasize that this innovative technology offers the ultimate benefit of being accessible to small producers in developing countries. Even though the algorithm was developed specifically for the classification of cherries, a similar methodology can be adapted to the processing of other fruits, for which their colour can be used as a quality discrimination factor.

\section{References}

Abdelghafour F., Rosu R., Keresztes B., Germain C., Da Costa J.P. 2019. A Bayesian framework for joint structure and colour based pixel-wise classification of grapevine proximal images. Comput. Electron. Agric. 158:345-57.

Ahmad I.S., Reid J.F.1996. Evaluation of colour representations for maize images. J. Agric. Eng. Res. 63:185-96.

Antonelli A., Cocchi M., Fava P., Foca G., Franchini G.C., Manzini D., Ulrici A. 2004. Automated evaluation of food colour by means of multivariate image analysis coupled to a wavelet-based classification algorithm. Analyt. Chim. Acta 515:3-13.

Arivazhagan S., Shebiah R.N., Nidhyanandhan S.S., Ganesan L. 2010. Fruit recognition using colour and texture features. Inf. Sci. 1:90-4.

Blasco J., Aleixos N., Moltó E. 2003. Machine vision system for automatic quality grading of fruit. Biosyst. Engine. 85:415-23.

Correa C., Valero C., Barreiro P., Diago M.P., Tardaguila J. 2012. Feature extraction on vineyard by Gustafson Kessel FCM and K-means. pp 481-484 in Electrotechnical Conference (MELECON), 16 $6^{\text {th }}$ IEEE Mediterranean, 25-28 March 2012.

Ebner M. 2004. A parallel algorithm for colour constancy. J. Parallel Distrib. Comput. 64:79-88.

Funck J.W., Zhong Y., Butler D.A., Brunner C.C., Forrer J.B. 2003. Image segmentation algorithms applied to wood defect detection. Comput. Electron. Agric. 41:157-79.

Gan H., Lee W.S., Alchanatis V., Ehsani R., Schueller J.K. 2018. Immature green citrus fruit detection using colour and thermal images. Comput. Electron. Agric. 152:117-25.

González R.C., Woods R.E. 2006. Tratamiento digital de imágenes. Addison-Wesley Longman, USA, pp. 800.

Kang H., Chen C. 2020. Fruit detection, segmentation and 3D visualisation of environments in apple orchards. Comput. Electron. Agric. 171:105302.

Khurram H., Chai D., Rassau A. 2018. A comprehensive review of fruit and vegetable classification techniques. Image Vision
Comput. 80:24-44.

Kondo N. 2010. Automation on fruit and vegetable grading system and food traceability. Trends Food Sci. Technol. 21:145-52.

Kotsiantis S.B. 2007. Supervised machine learning: a review of classification techniques. Informatica 31:249-68.

Lee D.J., Schoenberger R., Archibald J., McCollum S. 2008. Development of a machine vision system for automatic date grading using digital reflective near-infrared imaging. J. Food Engine. 86:388-98.

Leemans V., Magein H., Destain M.F. 2002. On-line fruit grading according to their external quality using machine vision. Biosyst. Engine. 83:397-404.

Momeny M., Jahanbakhshi A., Jafarnezhad K., Zhang Y.D. 2020. Accurate classification of cherry fruit using deep CNN based on hybrid pooling approach. Postharvest Biol. Technol. 166:111204.

Moreda G.P., Ortiz-Cañavate J., García-Ramos F.J., Ruiz-Altisent M. 2009. Non-destructive technologies for fruit and vegetable size determination - A review. J. Food Engine. 92:119-36.

Paulsson N., Stocklassa B. 1999. A real-time colour image processing system for forensic fiber investigations. Foren. Sci. Int. 103:37-59.

Paulus I., De Busscher R., Schrevens E. 1997. Use of image analysis to investigate human quality classification of apples. J. Agric. Eng. Res. 68:341-53.

Rahimizadeh H., Marhaban M.H., Kamil R.M., Ismail N.B. 2009. Colour image segmentation based on bayesian theorem and kernel density estimation. Eur. J. Sci. Res. 26:430-6.

Rungpichayapicheta P., Mahayotheeb B., Naglea M., Khuwijitjarub P., Müllera J. 2016. Robust NIRS models for non-destructive prediction of postharvest fruit ripeness and quality in mango, Postharv. Biol. Technol. 11:31-40.

Singh N., Delwiche M.J., Scott R. 1993. Image analysis methods for real-time colour grading of stone fruit. Comput. Electron. Agric. 9:71-84.

Sowmya B., Sheelarani B. 2009. Colour image segmentation using soft computing techniques. Int. J. Soft Comput. Appl. 4:69-80.

Tan K., Suk Lee W., Gan H., Wang Sh. 2018. Recognizing blueberry fruit of different maturity using histogram-oriented gradients and colour features in outdoor scenes. Biosyst. Engine. 176:59-72.

Wu G., Zhu Q., Huang M., Guo Y., Qin J. 2019. Automatic recognition of juicy peaches on trees based on $3 \mathrm{D}$ contour features and colour data. Biosyst. Engine. 188:1-13.

Zhang Ch., Guo Ch., Liu F., Kong W., He Y., Lou B. 2016. Hyperspectral imaging analysis for ripeness evaluation of strawberry with support vector machine. J. Food Engine. $179: 11-8$ 